\title{
ZAPPING SMALL FILTERS
}

\author{
CLAUDE LAFLAMME
}

(Communicated by Andreas R. Blass)

\begin{abstract}
We show two methods for diagonalizing filters of the form $F_{\sigma}$, first without adding an unbounded real, the other while preserving $P$-points; the interest lies in an attempt at destroying maximal almost disjoint families with least damage.
\end{abstract}

\section{INTRODUCTION}

Our aim is to investigate how to diagonalize, or zap filters on the natural numbers with the least harm done. Indeed, a natural approach to several open questions on "cardinal invariants of the continuum" such as the consistency of the inequalities $\mathfrak{d}<\mathfrak{a}, \mathfrak{i}<\mathfrak{a}, \mathfrak{u}<\mathfrak{a}$ (see [6]), is to iteratively destroy maximal almost disjoint families while preserving the appropriate objects. Obviously, the only way (up to equivalence) to destroy a maximal almost disjoint family $\mathscr{A}$ is to diagonalize the filter $\mathscr{F}(\mathscr{A})$ generated by the collection $\left\{A^{c}: A \in \mathscr{A}\right\}$.

We first develop a necessary condition for filters to be diagonalized in a forcing extension without adding an unbounded real, and then we verify that filters of the form $\mathscr{F}(\mathscr{A})$ do satisfy the condition. We only succeed however, in diagonalizing filters of the form $F_{\sigma}$ without adding an unbounded real. In the following section, we indicate how to diagonalize $F_{\sigma}$ while preserving $P$-points from the ground model.

This of course allows us also to diagonalize any filter that is included in an $F_{\sigma}$ filter while either preserving $P$-points or not adding an unbounded real. We finally show in the last section how to build in ZFC maximal almost disjoint families $\mathscr{A}$ for which $\mathscr{F}(\mathscr{A})$ is included in an $F_{\sigma}$ filter; however, the Continuum Hypothesis allows us to build an $\mathscr{A}$ such that $\mathscr{F}(\mathscr{A})$ is not included in any $F_{\sigma}$ filter and hence we probably have provided only a very slim partial result on the consistency of either $\mathfrak{d}<\mathfrak{a}$ or $\mathfrak{u}<\mathfrak{a}$.

We now briefly review some terminology.

We write $\omega$ for the set of natural numbers, $[\omega]^{\omega}$ for the set of its infinite subsets, and $\omega^{\omega}$ for the set of functions on the natural numbers. $X \subseteq^{*} Y$ means that $X \backslash Y$ is finite, and $X={ }^{*} Y$ means that $X \backslash Y \cup Y \backslash X$ is finite. A natural number $k$ is often identified with the set of its predecessors $\{0, \ldots$, $k-1\}$.

Received by the editors April 2, 1990 and, in revised form, August 27, 1990.

1980 Mathematics Subject Classification (1985 Revision). Primary 04A20; Secondary 03E35.

Supported by an NSERC University Research Fellowship. 
A filter $\mathscr{F}$ is a family of subsets of $\omega$ closed under supersets and finite intersections; we assume that all our filters consist only of infinite sets and contain the Fréchet filter $\mathscr{F} r$ of all cofinite sets. More generally, a collection of infinite sets is said to have the finite intersection property if the intersection of any finite subcollection is infinite. A set $X \in[\omega]^{\omega}$ is said to diagonalize, or to zap, a filter $\mathscr{F}$, if $X \subseteq \subseteq^{*} Y$ for each $Y \in \mathscr{F}$. A family $\mathscr{A} \subseteq[\omega]^{\omega}$ is almost disjoint if the pairwise intersection of (distinct) members of $\mathscr{A}$ is finite. We will also identify subsets of $\omega$ with their characteristic functions in $2^{\omega}$, the latter space being equipped with the product topology of the discrete topology on $2=\{0,1\}$.

Given two models $\mathscr{M} \subseteq \mathscr{N}$, we say that $f \in\left(\omega^{\omega}\right)^{\mathscr{N}}$ is unbounded if

$$
\left(\forall g \in\left(\omega^{\omega}\right)^{\mathscr{M}}\right)\left(\exists^{\infty} n\right)[f(n) \geq g(n)]
$$

and dominating if

$$
\left(\forall g \in\left(\omega^{\omega}\right)^{\mathscr{M}}\right)\left(\forall^{\infty} n\right)[f(n) \geq g(n)] .
$$

Here $\exists^{\infty} n$ means "there are infinitely many" and $\forall^{\infty} n$ means "for all but finitely many $n . "$ An ultrafilter $\mathscr{U} \in \mathscr{M}$ is said to be preserved if, in $\mathscr{N}$, $\overline{\mathscr{U}}=\{X \subseteq \omega:(\exists Y \in \mathscr{U}) Y \subseteq X\}$ is an ultrafilter.

\section{A NECESSARY CONDITION}

We attack the problem of diagonalizing a filter $\mathscr{F}$ without adding an unbounded real. It is clear first of all that the enumerating functions of sets in $\mathscr{F}$ should form a bounded family, which turns out by a result of Talagrand [5] to be equivalent to $\mathscr{F}$ being meager. Of course, diagonalizing $\mathscr{F}$ also zaps the filters $f(\mathscr{F})=\left\{X: f^{-1}(X) \in \mathscr{F}\right\}$ and hence the same should be true for those filters; this means that $\mathscr{F}$ is required to be "completely meager" (see [4]). Consider further the following situation: think of $\mathscr{F}$ as a filter on $\omega \times \omega$ and suppose it contains the set above the graph of each function in $\omega^{\omega}$; then diagonalizing $\mathscr{F}$ would actually add a dominating real.

Putting that together we get:

Proposition 2.1. If $\mathscr{F}$ can be diagonalized in a forcing extension without adding an unbounded real, then it is necessary that for each family $\left\langle X_{i}: i \in \omega\right\rangle$ such that:

1. Each $X_{i}$ is incompatible with $\mathscr{F}$, (that is $\left.X_{i}^{c} \in \mathscr{F}\right)$

2. $\bigcup_{i \in \omega} X_{i} \in \mathscr{F}$.

Then there exist $h \in \omega^{\omega}$ and a sequence $n_{0}<n_{1}<\cdots$ such that:

$$
(\forall Y \in \mathscr{F})\left(\forall^{\infty} k\right)\left[Y \cap \bigcup_{\left[n_{k}, n_{k+1}\right)} X_{i} \cap h(i) \neq \varnothing\right] .
$$

Proof. Suppose that some $Z \in[\omega]^{\omega}$ diagonalizes $\mathscr{F}$ in some forcing extension, and suppose that the condition fails for the family $\left\langle X_{i}: i \in \omega\right\rangle$ as above. We produce an unbounded real from $Z$. We consider two cases:

Case 1. (For all sequences $\left.n_{0}<n_{1}<\cdots\right)(\exists Y \in \mathscr{F})\left(\exists^{\infty} k\right)\left[Y \cap \bigcup_{i \in\left[n_{k}, n_{k+1}\right)} X_{i}\right.$ $=\varnothing]$.

This case corresponds to the situation when $h(\mathscr{F})$ is not meager, where $h(n)=$ the smallest $i$ such that $n \in X_{i}$. 
Define $\bar{Z}=\left\{i: X_{i} \cap Z \neq \varnothing\right\}$, which is infinite by the hypothesis on the family $\left\langle X_{i}: i \in \omega\right\rangle$, and put $f_{Z}(n)=n$th element of $\bar{Z}$. We claim that $f_{Z}$ is unbounded. Indeed fix $g \in\left(\omega^{\omega}\right)^{V}$, and define a sequence of natural numbers such that $n_{k+1}>g\left(n_{k}\right)$ for each $k$. By assumption we can find some $Y \in \mathscr{F}$ such that

hence for each such $k$, we get:

$$
\left(\exists^{\infty} k\right)\left[Y \cap \bigcup_{i \in\left[n_{k}, n_{k+1}\right)} X_{i}=\varnothing\right]
$$

$$
f_{Z}\left(n_{k}\right) \geq n_{k+1} \geq g\left(n_{k}\right)
$$

as desired.

Case 2. $\left(\exists n_{0}<n_{1}<\cdots\right)(\forall Y \in \mathscr{F})\left(\forall^{\infty} k\right)\left[Y \cap \bigcup_{i \in\left[n_{k}, n_{k+1}\right)} X_{i} \neq \varnothing\right]$.

Hence by assumption, it must be that:

$$
\left(\forall h \in \omega^{\omega}\right)(\exists Y \in \mathscr{F})\left(\exists^{\infty} k\right)\left[Y \cap \bigcup_{i \in\left[n_{k}, n_{k+1}\right)} X_{i} \cap h(i)=\varnothing\right] .
$$

Now define

$$
f_{Z}(k)=\min \left\{Z \cap \bigcup_{i \in\left[n_{k}, n_{k+1}\right)} X_{i}\right\} \text {. }
$$

Clearly, $f_{Z}$ is an unbounded function.

The first nontrivial example of filters satisfying the condition are filters of the form $\mathscr{F}(\mathscr{A})$ where $\mathscr{A}$ is a maximal almost disjoint family; they actually satisfy a slightly stronger condition.

Proposition 2.2. Let $\mathscr{A}$ be a maximal almost disjoint family and $\mathscr{F}=\mathscr{F}(\mathscr{A})$. Then for all families $\left\langle X_{i}: i \in \omega\right\rangle$ such that:

(1) Each $X_{i}$ is incompatible with $\mathscr{F}$,

(2) $\bigcup_{i \in \omega} X_{i}$ is compatible with $\mathscr{F}$.

Then there are $h \in \omega^{\omega}$ and a sequence $n_{0}<n_{1}<\cdots$ such that:

$$
(\forall Y \in \mathscr{F})\left(\forall^{\infty} k\right)\left[Y \cap \bigcup_{i \in\left[n_{k}, n_{k+1}\right)} X_{i} \cap h(i) \neq \varnothing\right] .
$$

Lemma 2.3. There are infinitely many $A \in \mathscr{A}$ such that $(\forall n)\left[A \cap \bigcup_{i>n} X_{i} \neq \varnothing\right]$. Proof. Assume that there are only finitely many such sets $A$, say $\left\{A_{i}: i<l\right\}$. By induction, try to build $C=\left\{c_{n}: n \in \omega\right\}$ such that

$$
c_{n} \in \bigcup_{i>n} X_{i} \backslash \bigcup_{i<l} A_{i} \text {. }
$$

If we succeed and $C$ is infinite, then the family $\mathscr{A} \cup\{C\}$ would contradict the maximality of $\mathscr{A}$ since it is almost disjoint. Hence $C$ must be finite and

$$
\bigcup_{i>n} X_{i} \subseteq^{*} \bigcup_{i<l} A_{i} \text { for some } n \text {. }
$$

This means that the set $\left(\bigcup_{i>n} X_{i}\right)^{c}$ belongs to $\mathscr{F}$ and hence that $\bigcup_{i<n} X_{i}$ is compatible with $\mathscr{F}$, a contradiction. 
Now to prove the proposition, fix a family $\left\{A_{i}: i \in \omega\right\}$ as in the lemma, and define a function $h \in \omega^{\omega}$ and a sequence $n_{0}<n_{1}<\cdots$ such that

$$
(\forall j \leq k) \bigcup_{i \in\left[n_{k}, n_{k+1}\right)} X_{i} \cap h(i) \cap A_{j} \neq \varnothing .
$$

We claim that this works. Indeed, suppose $Y \in \mathscr{F}$, say $Y={ }^{*} C_{0}^{c} \cap \cdots \cap C_{r}^{c}$, where $C_{i} \in \mathscr{A}$, and that

$$
\left(\exists^{\infty} k\right)\left[Y \cap \bigcup_{i \in\left[n_{k}, n_{k+1}\right)} X_{i} \cap h(i)=\varnothing\right]
$$

Then

$$
\left(\exists^{\infty} k\right) \bigcup_{i \in\left[n_{k}, n_{k+1}\right)} X_{i} \cap h(i) \subseteq C_{0} \cup \cdots \cup C_{r} .
$$

But this means that $A_{j} \cap \bigcup_{i \leq r} C_{i}$ is infinite for each $j$, and hence is a contradiction.

The second example consists of the filters of the form $F_{\sigma}$, that is filters that can be written as a countable union of closed sets. The Fréchet filter is such a filter, since $\mathscr{F} r=\bigcup_{i \in \omega} C_{i}$ where $C_{i}=\left\{X \subseteq \omega\right.$ : the size of $X^{c}$ is at most $\left.i\right\}$ is a closed set.

The following, taken from [4], shows that all the information regarding a $F_{\sigma}$ filter is contained in a closed set.

Lemma 2.4. If $\mathscr{F}$ is an $F_{\sigma}$ filter, then there is a closed set $C$ with the finite intersection property which generates $\mathscr{F}$ (i.e., $\left.(\forall X \in \mathscr{F})(\exists Y \in C)\left[Y \subseteq^{*} X\right]\right)$. Proof. If $\mathscr{F}=\bigcup_{i \in \omega} C_{i}$, then let $C=\left\{X \cup i: X \in C_{i}\right\}$.

Proposition 2.5. Let $\mathscr{F}$ be an $F_{\sigma}$ filter and $\left\langle X_{i}: i \in \omega\right\rangle$ be a family such that:

(1) Each $X_{i}$ is incompatible with $\mathscr{F}$,

(2) $\bigcup_{i \in \omega} X_{i}$ is compatible with $\mathscr{F}$.

Then there are $h \in \omega^{\omega}$ and a sequence $n_{0}<n_{1}<\cdots$ such that:

$$
(\forall Y \in \mathscr{F})\left(\forall^{\infty} k\right)\left[Y \cap \bigcup_{i \in\left[n_{k}, n_{k+1}\right)} X_{i} \cap h(i) \neq \varnothing\right] .
$$

Proof. Fix a closed set $C$ which generates $\mathscr{F}$ as in the lemma. Proceeding by induction, suppose that we have already defined $n_{k}$ and $h \uparrow n_{k}$. Let $n=$ $h\left(n_{k}-1\right)$ and $X=\bigcup_{i \geq n_{k}} X_{i}$, which is compatible with $\mathscr{F}$ by assumption. Then $C(X)=\{Y \cap X: Y \in C\}$ is a closed set with the finite intersection property, and hence we can find $m>n$ such that $Z \cap[n, m) \neq \varnothing$ for each $Z \in C(X)$. Now choose $n_{k+1}$ large enough so that

$$
(\forall Y \in C) Y \cap[n, m) \cap \bigcup_{i \in\left[n_{k}, n_{k+1}\right)} X_{i} \neq \varnothing
$$

and put $h\left\lceil\left[n_{k}, n_{k+1}\right)=m\right.$.

In the next section we show how to diagonalize $F_{\sigma}$ filters without adding an unbounded real. It is more difficult to find a reasonable necessary condition for a filter to be diagonalizable in a forcing extension while preserving some 
ultrafilters. However, since the enumerating functions of sets in an ultrafilter always form an unbounded family, an obvious requirement is that we do not add a dominating real. It should be noticed that analytic filters do not in general satisfy the above necessary condition; indeed, diagonalizing an analytic filter will in general add a dominating real (see [4]).

\section{3. $F_{\sigma}$ FILTERS AND UNBOUNDED REALS}

We now show that we can diagonalize $F_{\sigma}$ filters without adding an unbounded real.

Theorem 3.1. It is possible to diagonalize any $F_{\sigma}$ filter in a forcing extension without adding an unbounded real.

First, we look in more detail at the structure of those filters.

Lemma 3.2. Let $F$ be an $F_{\sigma}$ filter. Then there are a sequence $n_{0}<n_{1}<\cdots$ and sets $a_{0}^{k}, \ldots, a_{r_{k}-1}^{k} \subseteq\left[n_{k}, n_{k+1}\right)$ for each $k$ such that:

(1) $\left(\forall x \in\left[r_{k}\right]^{k}\right) \bigcap_{i \in x} a_{i}^{k} \neq \varnothing$,

(2) $(\forall X \in \mathscr{F})\left(\exists f \in \prod_{k \in \omega} r_{k}\right)\left[\bigcup\left\{a_{f(k)}^{k}: k \in \omega\right\} \subseteq^{*} X\right]$.

i.e., $\mathscr{F}$ is included in the $\left(F_{\sigma}\right)$ filter generated by the sets $\left\{X:(\forall k)\left(\exists i<r_{k}\right) a_{i}^{k} \subseteq\right.$ $X\}$.

Proof. As in Lemma 2.4, let $C$ be a closed set with the finite intersection property which generates $\mathscr{F}$. Given $n_{k}$, choose $n_{k+1}$ large enough so that

$$
\left(\forall X_{1}, \ldots, X_{k} \in C\right)\left[X_{1} \cap \cdots \cap X_{k} \cap\left[n_{k}, n_{k+1}\right) \neq \varnothing\right] .
$$

Now list $\left\{X \cap\left[n_{k}, n_{k+1}\right): X \in C\right\}$ as $a_{0}^{k}, \ldots, a_{r_{k}-1}^{k}$. This completes the proof.

According to the lemma, we have reduced the problem of zapping $F_{\sigma}$ filters to the following problem:

Given $r_{k} \in \omega$, for $k \in \omega$, forcing $x_{k} \in\left[r_{k}\right]^{k}, k \in \omega$, such that

$$
\left(\forall f \in \prod_{k \in \omega} r_{k}\right)^{V}\left(\forall^{\infty} k\right)\left[f(k) \in x_{k}\right],
$$

and without adding an unbounded real. Indeed, given the family $\left\langle x_{k}: k \in \omega\right\rangle$ as above, the set $Z=\left\{z_{k}: k \in \omega\right\}$ where $z_{k} \in \bigcap_{i \in x_{k}} a_{i}^{k}(\neq \varnothing)$, will diagonalize the filter $\mathscr{F}$.

Although some specific cases can be dealt with simply by adding a random real (when $r_{k}$ is not too large compared to $k$ ), the essential idea of the general case is due to Shelah [1] (i.e., forcing with perfect trees that are finitely but "profusely" branching does not add an unbounded real).

First let $s_{k}=\left(\begin{array}{c}r_{k} \\ k\end{array}\right)$ and list $\left[r_{k}\right]^{k}=\left\{x_{0}^{k}, \ldots, x_{s_{k}-1}^{k}\right\}$. Define

$$
\begin{aligned}
& \mathbb{P}=\left\{T: T \text { is a perfect tree of } \bigcup_{n} \prod_{k<n} s_{k}\right. \text { such that } \\
& \qquad \begin{aligned}
(\forall n)(\exists \bar{m})(\forall m \geq \bar{m})\left(\forall a_{1}, \ldots, a_{n} \in r_{m}\right)\left(\forall s \in \omega^{m} \cap T\right) \\
\left.\left|\left\{t \in \operatorname{succ}_{T}(s):\left\{a_{1}, \ldots, a_{n}\right\} \subseteq x_{t(m)}^{m}\right\}\right| \geq n\right\}
\end{aligned}
\end{aligned}
$$

with ordering being inclusion. 
As usual, for $s \in T, T_{s}$ will denote the collection of nodes from $T$ that are comparable with $s$.

Claim 3.3. If $G$ is $\mathbb{P}$-generic and $g=\bigcap_{T \in G} T$, then

$$
\left(\forall f \in \prod_{k \in \omega} r_{k}\right)^{V}\left(\forall^{\infty} k\right)\left[f(k) \in x_{g(k)}^{k}\right]
$$

as desired, i.e., the desired $x_{k}$ is $x_{g(k)}^{k}$.

Proof. Given $f \in \prod_{k \in \omega} r_{k}$ which belongs to the ground model $V$, it suffices to verify that

$$
\Delta(f)=\left\{T \in \mathbb{P}:(\exists \bar{m})(\forall m \geq \bar{m})\left(\forall s \in \omega^{m+1} \cap T\right) f(m) \in x_{s(m)}^{m}\right\}
$$

is dense. Now given $T \in \mathbb{P}$, choose $\bar{m}$ such that

$$
\begin{aligned}
& (\forall m \geq \bar{m})\left(\forall a_{1}, a_{2} \in r_{m}\right)\left(\forall s \in \omega^{m} \cap T\right) \\
& \left|\left\{t \in \operatorname{succ}_{T}(s):\left\{a_{1}, a_{2}\right\} \subseteq x_{t(m)}^{m}\right\}\right| \geq 2 ;
\end{aligned}
$$

then $T^{\prime}=T \cap\left\{s:(\forall m \geq \bar{m}) f(m) \in x_{s(m)}^{m}\right\} \in \Delta(f)$ as desired.

We only need to show that $\mathbb{P}$ adds no unbounded real.

Definition 3.4. For $k \in \omega$, we define:

$$
\begin{aligned}
& T^{\prime} \leq_{k} T \quad \text { if }(\forall m)\left(\forall s \in \omega^{m} \cap T\right) \operatorname{succ}_{T^{\prime}}(s) \neq \operatorname{succ}_{T}(s) \Rightarrow \\
&\left(\forall a_{1}, \ldots, a_{k} \in r_{m}\right)\left|\left\{t \in \operatorname{succ}_{T^{\prime}}(s):\left\{a_{1}, \ldots, a_{k}\right\} \subseteq x_{t(m)}^{m}\right\}\right| \geq k .
\end{aligned}
$$

Observe that if $T^{\prime \prime} \leq_{k^{\prime}} T^{\prime} \leq_{k} T$ and $k \leq k^{\prime}$, then $T^{\prime \prime} \leq_{k} T$.

Lemma 3.5. Suppose that $T^{n+1} \leq_{k_{n}} T^{n}$ for $n \in \omega$, where $\left\langle k_{n}: n \in \omega\right\rangle$ is an increasing sequence. Then

$$
T=\bigcap T^{n} \in \mathbb{P} \text { and } T \leq_{k_{n}} T^{n} \text { for each } n .
$$

Proof. First show by induction on the levels that $T$ must be a perfect tree; we show that $T \in \mathbb{P}$.

Fix $n \in \omega$, we need to find $\bar{m}$ such that:

$$
\begin{aligned}
& (\forall m \geq \bar{m})\left(\forall a_{1}, \ldots, a_{n} \in r_{m}\right)\left(\forall s \in \omega^{m} \cap T\right) \\
& \left|\left\{t \in \operatorname{succ}_{T}(s):\left\{a_{1}, \ldots, a_{n}\right\} \subseteq x_{t(m)}^{m}\right\}\right| \geq n .
\end{aligned}
$$

Choose $\bar{n}$ such that $k_{\bar{n}}>n$ and fix $\bar{m}$ such that

$$
\begin{gathered}
(\forall m \geq \bar{m})\left(\forall s \in \omega^{m} \cap T^{\bar{n}}\right)\left(\forall a_{1}, \ldots, a_{n} \in r_{m}\right) \\
\left|\left\{t \in \operatorname{succ}_{T^{\bar{n}}}(s):\left\{a_{1}, \ldots, a_{n}\right\} \subseteq x_{t(m)}^{m}\right\}\right| \geq n .
\end{gathered}
$$

We claim that $\bar{m}$ works; indeed fix $m \geq \bar{m}, s \in \omega^{m} \cap T$, and $a_{1}, \ldots, a_{n} \in r_{m}$. Now $s \in T^{\bar{n}}$ but we can also find $\overline{\bar{n}} \geq \bar{n}$ such that $\operatorname{succ}_{T}(s)=\operatorname{succ}_{T^{\bar{n}}}(s)$; but the result follows since $T^{\overline{\bar{n}}} \geq_{k_{\bar{n}}} T^{\bar{n}}$.

Lemma 3.6. Assume that $T \Vdash \tau \in \omega$ and let $k \in \omega$. Then there are $T^{\prime} \leq_{k} T$, $n \in \omega$, such that

$$
\left(\forall s \in \omega^{n} \cap T^{\prime}\right)\left(\exists a_{s} \in \omega\right)\left[T_{s}^{\prime} \Vdash \tau=a_{s}\right] .
$$


Proof. Let

$$
S=\left\{t \in T:\left(\exists n_{t} \in \omega\right)\left(\exists T^{\prime} \leq_{k} T_{t}\right)\left(\forall s \in T^{\prime} \cap \omega^{n_{t}}\right)\left(\exists a_{s} \in \omega\right) T_{s}^{\prime} \Vdash \tau=a_{s}\right\} .
$$

Choose $\bar{m}$ such that

$$
\begin{aligned}
& (\forall m \geq \bar{m})\left(\forall s \in \omega^{m} \cap T\right)\left(\forall a_{1}, \ldots, a_{2 k} \in r_{m}\right) \\
& \left|\left\{t \in \operatorname{succ}_{T}(s):\left\{a_{1}, \ldots, a_{2 k}\right\} \subseteq x_{t(m)}^{m}\right\}\right| \geq 2 k .
\end{aligned}
$$

It suffices to show that $T \cap \omega^{\bar{m}} \subseteq S$. Indeed, if we have $T(t) \leq_{k} T_{t}$ for each $t \in \omega^{\bar{m}} \cap T \subseteq S$ with associated $n_{t}$, then $T^{\prime}=\bigcup_{t \in \omega^{\bar{m}} \cap T} T(t) \leq_{k} T$ and $n=\max \left\{n_{t}: t \in \omega^{\bar{m}} \cap T\right\}$ fulfills the requirements of the lemma.

Hence, fix $s \in \omega^{\bar{m}} \cap T$, and suppose for the sake of a contradiction that $s \notin S$. First of all, observe that

$$
\begin{aligned}
& (\forall m)\left(\forall t \in \omega^{m} \cap T\right) t \notin S \Rightarrow\left(\exists a_{1}, \ldots, a_{k} \in r_{m}\right) \\
& \left|\left\{t^{\prime} \in \operatorname{succ}_{T}(t) \cap S:\left\{a_{1}, \ldots, a_{k}\right\} \subseteq b_{t^{\prime}(m)}^{m}\right\}\right|<k .
\end{aligned}
$$

We shall build $T^{\prime} \leq_{k} T_{s}$ such that

$$
(\vee m \geq \bar{m})\left[T^{\prime} \cap S \cap \omega^{m}=\varnothing\right] .
$$

This yields the desired contradiction since we can now find $T^{\prime \prime} \leq T^{\prime}$ and $n \in \omega$ such that $T^{\prime \prime} \Vdash \tau=n$; finally choosing $t \in T$ and $T^{\prime \prime \prime} \leq T^{\prime \prime}$ such that $T^{\prime \prime \prime} \leq_{k} T_{t}$ shows that $t \in S$ !

Let $T^{\prime}=\{t \in T: t \leq s$ or $t \geq s$ and $t \notin S\} . T^{\prime}$ is a perfect tree; we show that $T^{\prime} \leq_{k} T_{s}$ and $T^{\prime} \in \mathbb{P}$ under our assumption that $s \notin S$.

To verify that $T^{\prime} \leq_{k} T_{s}$, fix $t \in \omega^{m} \cap T^{\prime}$ such that $\operatorname{succ}_{T^{\prime}}(t) \neq \operatorname{succ}_{T_{s}}(t)$. Since $t \notin S$, there are $a_{1}^{\prime}, \ldots, a_{k}^{\prime} \in r_{m}$ such that

$$
\left|\left\{t^{\prime} \in \operatorname{succ}_{T}(t) \cap S:\left\{a_{1}^{\prime}, \ldots, a_{k}^{\prime}\right\} \subseteq x_{t^{\prime}(m)}^{m}\right\}\right|<k .
$$

But by assumption, since $t \in \omega^{m}$ and $m \geq \bar{m}$, we have that for all $a_{1}, \ldots, a_{2 k}$ $\in r_{m}$,

$$
\left|\left\{t^{\prime} \in \operatorname{succ}_{T}(t):\left\{a_{1}, \ldots, a_{2 k}\right\} \subseteq x_{t^{\prime}(m)}^{m}\right\}\right| \geq 2 k
$$

and hence, for all $a_{1}, \ldots, a_{k} \in r_{m}$,

$$
\left|\left\{t^{\prime} \in \operatorname{succ}_{T}(t) \backslash S:\left\{a_{1}, \ldots, a_{k}\right\} \subseteq x_{t^{\prime}(m)}^{m}\right\}\right| \geq 2 k-k=k
$$

as desired.

It remains to show that $T^{\prime} \in \mathbb{P}$; so we fix $n \in \omega$, and we show how to find $\bar{l}$ such that:

$$
\begin{gathered}
(\forall l \geq \bar{l})\left(\forall a_{1}, \ldots, a_{n} \in r_{l}\right)\left(\forall t \in \omega^{l} \cap T^{\prime}\right) \\
\left|\left\{t^{\prime} \in \operatorname{succ}_{T^{\prime}}(t):\left\{a_{1}, \ldots, a_{n}\right\} \subseteq x_{t^{\prime}(l)}^{l}\right\}\right| \geq n .
\end{gathered}
$$

Without loss of generality we assume that $n \geq k$. Since $T_{s} \in \mathbb{P}$, we have $\bar{l}$ such that

$$
\begin{gathered}
(\forall l \geq \bar{l})\left(\forall t \in \omega^{l} \cap T_{s}\right)\left(\forall a_{1}, \ldots, a_{2 n} \in r_{l}\right) \\
\left|\left\{t^{\prime} \in \operatorname{succ}_{T_{s}}(t):\left\{a_{1}, \ldots, a_{2 n}\right\} \subseteq x_{t^{\prime}(l)}^{l}\right\}\right| \geq 2 n .
\end{gathered}
$$

Now for all $t \in \omega^{l} \cap T^{\prime}$, since again $t \notin S$, there are $a_{1}^{\prime}, \ldots, a_{k}^{\prime} \in r_{l}$ such that

$$
\left|\left\{t^{\prime} \in \operatorname{succ}_{T}(t) \cap S:\left\{a_{1}^{\prime}, \ldots, a_{k}^{\prime}\right\} \subseteq x_{t^{\prime}(l)}^{l}\right\}\right|<k,
$$


and we then have for all $a_{1}, \ldots, a_{n} \in r_{l}$,

$$
\left|\left\{t^{\prime} \in \operatorname{succ}_{T}(t) \backslash S:\left\{a_{1}, \ldots, a_{n}\right\} \subseteq x_{t^{\prime}(l)}^{l}\right\}\right| \geq 2 n-k \geq n
$$

as desired.

We finally verify that $\mathbb{P}$ adds no unbounded real. Suppose now that $T \Vdash$ $\dot{f} \in \omega^{\omega}$. Build $\left\langle T^{n}: n \in \omega\right\rangle$ and $\left\langle k_{n}: n \in \omega\right\rangle$ such that $T^{n+1} \leq_{k_{n}} T^{n}$ and such that

$$
\left(\forall s \in \omega^{k_{n}} \cap T^{n}\right)\left(\exists a_{s} \in \omega\right)\left[T_{s}^{n} \Vdash \dot{f}(n+1)=a_{s}\right] .
$$

Then $T=\bigcap_{n} T^{n} \in \mathbb{P}$ and if $g(n)=\max \left\{a_{s}: s \in \omega^{k_{n}} \cap T\right\}$, then $T \Vdash(\forall n) \dot{f}(n) \leq$ $g(n)$ as desired.

\section{4. $F_{\sigma}$ FILTERS AND $P$-POINTS}

We now show how to diagonalize $F_{\sigma}$ filters while preserving $P$-points from the ground model.

Theorem 4.1. It is possible to diagonalize any $F_{\sigma}$ filter in a forcing extension while preserving all P-points from the ground model.

The proof uses the setup of $\S 3$, and follows almost identically the construction of the partial order $Q$ from $\S 2$ of the Blass-Shelah model [2]. Indeed, we define our partial order so closely to theirs that almost all required lemmas can be copied without change; we will only guide the reader through the very few necessary modifications.

So let us fix $\mathscr{F}$ an $F_{\sigma}$ filter; as in Lemma 3.2, there are a sequence $n_{0}<$ $n_{1}<\cdots$ and sets $a_{0}^{k}, \ldots, a_{r_{k}-1}^{k} \subseteq\left[n_{k}, n_{k+1}\right)$ for each $k$ such that:

1. $\left(\forall x \in\left[r_{k}\right]^{k}\right) \bigcap_{i \in x} a_{i}^{k} \neq \varnothing$,

2. $(\forall X \in \mathscr{F})\left(\exists f \in \prod_{k \in \omega} r_{k}\right)\left[\bigcup\left\{a_{f(k)}^{k}: k \in \omega\right\} \subseteq^{*} X\right]$.

It thus suffices, in order to diagonalize $\mathscr{F}$, to force $Y \in[\omega]^{\omega}$ and $x_{k} \in\left[r_{k}\right]^{k}$, for $k \in Y$, such that:

$$
\left(\forall f \in \prod_{k \in \omega} r_{k}\right)^{V}\left(\forall^{\infty} k \in Y\right)\left[f(k) \in x_{k}\right] .
$$

Notice that in $\S 3$, we obtained $Y=\omega$, but the weakening here seems necessary.

We can assume without loss of generality that $r_{k}>k$. Let $s_{k}=\left(\begin{array}{c}r_{k} \\ k\end{array}\right)$ and list $\left[r_{k}\right]^{k}=\left\{x_{0}^{k}, \ldots, x_{s_{k}-1}^{k}\right\}$.

Define $\pi_{k}=\bigcup\left\{\prod_{i \in y} s_{i}: \varnothing \neq y \subseteq k\right\}$ and for $n<m$, let $K_{n, m}$ be the set of binary relations $t \subseteq \pi_{n} \times \pi_{m}$ such that, for each $a \in \pi_{n}$,

$$
(a, a) \in t \text {, and if }(a, b) \in t \text {, then } b\lceil n=a \text {. }
$$

For $t \in K_{n, m}$, we define the depth $\mathrm{Dp}(t)$ by the following induction:

$\operatorname{Dp}(t) \geq 0$ always.

$\operatorname{Dp}(t) \geq 1$ if, for each $a \in \pi_{n}$, there is $b \in \pi_{m}$ such that $(a, b) \in t$ and $b \neq a$.

$\operatorname{Dp}(t) \geq d+1$, for $d \geq 1$, if, for each $f \in \prod_{i<m} r_{i}$,

$$
t_{f}:=\left\{(a, b) \in t:(\forall i \in \operatorname{dom}(b \backslash a)) f(i) \in x_{b(i)}^{i}\right\}
$$

has depth $\geq d$. 
Equivalently, $t$ has depth $\geq d+1$ iff

$$
\left(\forall f_{1}, \ldots, f_{d} \in \prod_{i \in \omega} r_{i}\right)\left(\forall a \in \pi_{n}\right)\left(\exists b \neq a \text { in } \pi_{m}\right)\left[(a, b) \in \bigcap_{i=1, \ldots, d} t_{f_{i}}\right] .
$$

Equipped with a copy of [2], the reader will have noticed the similarities; in particular, $\pi_{n}$ here plays the role of $\wp(n)$ there. So the partial order $Q$ is defined similarly as the collection of all pairs $\langle w, T\rangle$ such that $T=\left\langle t_{l}: l \in \omega\right\rangle$ and for some increasing function $n: \omega \rightarrow \omega$ we have:

(1) $w \in \pi_{n(0)}$,

(2) $t_{l} \in K_{n(l), n(l+1)}$ for each $l$, and

(3) $\mathrm{Dp}\left(t_{l}\right) \rightarrow \infty$ as $l \rightarrow \infty$.

Another such condition $\left\langle w^{\prime}, T^{\prime}\right\rangle$ is an extension of $\langle w, T\rangle$ if and only if there is an increasing function $k: \omega \rightarrow \omega$ such that, writing $t_{l}^{*}$ for the usual composition of relations $t_{k(l)} t_{k(l)+1} \cdots t_{k(l+1)-1}$, we have:

(1) $\left\langle w, w^{\prime}\right\rangle \in t_{0} t_{1} \cdots t_{k(0)-1}$,

(2) $t_{l}^{\prime} \in K_{n(k(l)), n(k(l+1))}$ for all $l \in \omega$, and

(3) $t_{l}^{\prime} \subseteq t_{l}^{*}$ for all $l \in \omega$.

We show that forcing with $Q$ does diagonalize our filter.

Lemma 4.2. If $G$ is Q-generic, then $g=\bigcup\{w:\langle w, T\rangle \in G\} \in \prod_{k \in Y} s_{k}$ for some $Y \in[\omega]^{\omega}$, and further,

$$
\left(\forall f \in \prod_{k \in \omega} r_{k}\right)^{V}\left(\forall^{\infty} k \in Y\right)\left[f(k) \in x_{g(k)}^{k}\right] .
$$

Proof. Let $f \in\left(\prod_{k \in \omega} r_{k}\right)^{V}$ and $\langle w, T\rangle \in Q$ be given, where $T=\left\langle t_{l}: l \in \omega\right\rangle$. Define $t_{l}^{\prime}=\left(t_{l}\right)_{f}$ and $T^{\prime}=\left\langle t_{l}^{\prime}: l \in \omega\right\rangle$; then $\left\langle w, T^{\prime}\right\rangle \leq\langle w, T\rangle$ belongs to $Q$ and forces that

$$
\left(\forall^{\infty} k \in Y\right)\left[f(k) \in x_{g(k)}^{k}\right]
$$

as desired.

The verification that $Q$ preserves the ground model $P$-points is essentially unchanged from [2], only easier.

\section{CONCLUSION}

We are now in position to diagonalize all filters that are included in an $F_{\sigma}$ filter without adding an unbounded real or while preserving $P$-points. This will be the case for some filters of the form $\mathscr{F}(\mathscr{A})$, in particular if there are $n_{0}<n_{1}<\cdots$ such that

$$
n_{k+1}-n_{k} \rightarrow \infty \text { as } k \rightarrow \infty \quad \text { and } \quad(\forall A \in \mathscr{A})\left(\forall^{\infty} k\right)\left[\left|A \cap\left[n_{k}, n_{k+1}\right)\right| \leq 1\right] .
$$

Such maximal almost disjoint families are easily constructed using Zorn's lemma by fixing a sequence $\left\langle n_{k}: k \in \omega\right\rangle$ as above and choosing $\mathscr{A}$ a maximal element of the partial order, under inclusion, of all almost disjoint families satisfying the above second condition. However, using the Continuum Hypothesis, we show that not all filters of the form $\mathscr{F}(\mathscr{A})$ are included in an $F_{\sigma}$ filter, and hence we feel that we have only grazed the problem. 
Proposition 5.1 $(\mathrm{CH})$. There is a maximal almost disjoint family $\mathscr{A}$ such that $\mathscr{F}(\mathscr{A})$ is not included in any $F_{\sigma}$ filter.

Proof. It suffices (and is equivalent) to build $\mathscr{A}$ such that for all closed sets $C$ which generate a proper filter,

$$
(\exists A \in \mathscr{A})(\forall X \in C) X \cap A \text { is infinite. }
$$

Indeed, if $\mathscr{F}(\mathscr{A}) \subseteq \mathscr{F}$, where $\mathscr{F}$ is an $F_{\sigma}$ filter, choose a closed set $C$ which generates $\mathscr{F}$; now our assumptions provide a contradiction.

Using $\mathrm{CH}$, we list all concerned closed sets in an $\omega_{1}$ sequence and we build the family $\mathscr{A}$ by induction. At stage $\alpha$, we already have defined countably many sets $\left\{A_{n}: n \in \omega\right\}$ from our almost disjoint family, and we built a new set $A$ almost disjoint from the $A_{n}$ 's and fulfilling our requirement for the $\alpha$ th closed set $C_{\alpha}$.

If $\mathscr{F}\left(\left\{A_{n}: n \in \omega\right\}\right)$ is not included in the filter generated by $C_{\alpha}$, then we have nothing to do. Otherwise, we ensure that:

$$
\left(\forall X \in C_{\alpha}\right) X \cap A \text { is infinite. }
$$

By induction, choose numbers $n_{k}<n_{k+1}, X_{k} \in C_{\alpha}$ such that

1. $X_{k} \subseteq^{*} A_{k}^{c}$,

2. $\left(\forall Y \in C_{\alpha}\right) Y \cap X_{0} \cap \cdots \cap X_{k} \cap\left[n_{k}, n_{k+1}\right) \neq \varnothing$.

This is possible by our assumptions. Finally let

$$
A=\bigcup_{k \in \omega}\left\{X_{0} \cap \cdots \cap X_{k} \cap\left[n_{k} n_{k+1}\right)\right\} .
$$

Note however that for any almost disjoint family $\mathscr{A}$ and $A \in \mathscr{A}$, the filter $\mathscr{F}^{\prime}=\langle\mathscr{A} \backslash\{A\}\rangle$ is included in an $F_{\sigma}$ filter since $A$ zaps $\mathscr{F}^{\prime}$; but adjoining $A^{c}$ can yield a filter, as above, which is not included in any $F_{\sigma}$ filter anymore.

One might have hoped that such (maximal) almost disjoint families with the above "strengthened" maximality condition would be forced to have size continuum; however, the standard "Kunen" trick (see [3, Theorem 2.3, p. 256]) allows one to build, using $\mathrm{CH}$, such a family that is indestructible by Cohen forcing. Indeed, they appear to be the likely candidates for small maximal almost disjoint families.

Added in proof. The first forcing also preserves $P$-points.

\section{REFERENCES}

1. S. Shelah, Vive la différence, no. 326, October 1990.

2. A. Blass and S. Shelah, There may be simple $P_{\aleph_{1}}$ and $P_{\aleph_{2}}$ points and the Rudin-Keisler ordering may be downward directed, Ann. Pure Appl. Logic 83 (1987), 213-243.

3. K. Kunen, Set theory, North-Holland, Amsterdam, 1980.

4. C. Laflamme, Forcing with filters and complete combinatorics, Ann. Pure Appl. Logic $\mathbf{4 2}$ (1989), 125-163.

5. M. Talagrand, Compacts de fonctions mesurables et filtres non mesurables, Studia Math. 67 (1980), 13-43.

6. J. Vaughan, Small uncountable cardinals and topology, preprint, 1989. 1 P3

Department of Mathematics, York University, North York, Ontario, Canada, M3J 